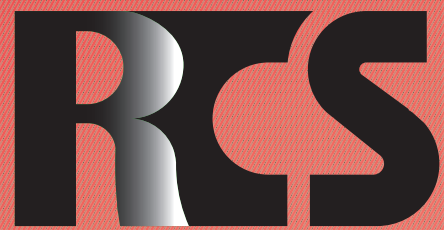

Depósito legal ppi $201502 Z U 4662$

Esta publicación científica en formato digital es continuidad de la revista impresa Depósito Legal: pp $197402 Z$ Z789

- ISSN: 1315-9518 • ISSN-E: 2477-9431

Universidad del Zulia. Revista de la Facultad de Ciencias Económicas y Sociales Vol. XXVI. No.1 


\title{
Gestión de ganancias en el riesgo de quiebra de las empresas públicas mexicanas
}

\author{
Cumpean Luna, Joel Alejandro* \\ Briseño García, Arturo** \\ Arango Herrera, Eduardo ${ }^{* * *}$
}

\section{Resumen}

Un fenómeno altamente estudiado dentro del entorno empresarial es la quiebra en las organizaciones. Sin embargo, un enfoque con el que ha sido poco relacionado dicho estatus es la calidad de la información financiera que reportan las empresas. De esta forma, el objetivo de la presente investigación es analizar la relación entre el riesgo de quiebra y la gestión de ganancias que practican las empresas públicas en México. Analizando un universo de 145 empresas y teniendo como muestra un total de 86 organizaciones que cotizaron en la Bolsa Mexicana de Valores durante el periodo 2014-2018, se desarrolló un estudio cuantitativo, longitudinal, y causal, recopilando información de fuentes secundarias como Bloomberg y reportes anuales de las empresas en sus sitios web. Se realizó una regresión panel de Mínimos Cuadrados Generalizados con apoyo de Stata 12. Los resultados arrojan que el incremento en la gestión de ganancias aumenta el riesgo de quiebra en las empresas de la muestra de este estudio. Se concluye que, al gestionar las ganancias de las empresas de la Bolsa Mexicana de Valores, se puede incrementar el riesgo de quiebra de las mismas y representar un grave problema para sus grupos de interés.

Palabras clave: Gestión de ganancias; riesgo de quiebra; mercados emergentes; empresas públicas mexicanas; estudio longitudinal.

Estudiante de doctorado en Ciencias Administrativas en la Universidad Autónoma de Tamaulipas, México. Maestro en Dirección Empresarial. E-mail: cpjoel.cumpean@gmail.com iD ORCID: https://orcid.org/0000-0002-5020-6854

** Doctor en Ciencias Administrativas. Profesor de Ética Corporativa en la Universidad Autónoma de Tamaulipas, México. Miembro de la Academia de Ciencias Administrativas y del Sistema Nacional de Investigadores (Nivel Candidato, 2019-2021). E-mail: abriseno@docentes.uat.edu.mx iD ORCID: https://orcid.org/0000-0002-6567$241 \mathrm{X}$

*** Doctor en Ciencias Administrativas. Profesor de Administración en la Universidad Autónoma de Tamaulipas, México. E-mail: dca.arango@gmail.com (iD ORCID: https://orcid.org/0000-0002-3780-6783 


\title{
Earnings management on the bankruptcy risk of Mexican public companies
}

\begin{abstract}
A highly studied phenomenon within the business environment is bankruptcy in organizations. However, an area in which this status has been less related is the quality of financial information reported by companies. Accordingly, the objective of this research is to analyze the relationship between bankruptcy risk and earnings management practiced by public companies in Mexico. Analyzing a universe of 145 companies and taking as a sample a total of 86 companies that were listed on the Mexican Stock Exchange during the 2014-2018 period, a quantitative, longitudinal, and causal study was developed, collecting data from secondary sources such as Bloomberg and annual reports of companies on their websites. A Generalized Least Squares regression panel was performed with the support of STATA 12. The results show that the increase in earnings management increases the risk of bankruptcy in the companies in the sample of this study. It is concluded that, when managing the earnings of the companies of the Mexican Stock Exchange, their risk of bankruptcy may increase and represent a serious problem for their stakeholders.
\end{abstract}

Keywords: Earnings management; bankruptcy risk; emerging markets; Mexican public companies; longitudinal study.

\section{Introducción}

Dentro del entorno empresarial, un fenómeno que ha sido fuertemente investigado debido a las grandes consecuencias que el mismo conlleva, es la quiebra de las empresas (Báez y Puentes, 2018; Martínez, et al., 2020). Bryan, Dinesh y Tripathy (2013), mencionan que este estatus acontece cuando una empresa es incapaz de dar cumplimiento a sus obligaciones financieras actuales y se presenta ante un tribunal federal para solicitar un periodo de recuperación y así poder reorganizar sus deudas o liquidar sus activos. Los efectos de la quiebra o bancarrota perjudican fuertemente tanto a la empresa que la sufre, como a diversos grupos de interés de la misma, entre ellos sus empleados, proveedores, acreedores y clientes (Rodríguez, Piñeiro y De Llano, 2014).

Para alertar a las organizaciones y a sus grupos de interés y así, tratar de evitar que las empresas lleguen a dicho estado de quiebra o bancarrota, se ha examinado el desempeño operativo y financiero de las mismas en los últimos 60 años. Sin embargo, pocos estudios han tomado en consideración variables como la discrecionalidad de la administración en los devengos para manipular el resultado de las empresas, puesto que dicha manipulación, mejor conocida como "gestión de ganancias", no es una situación observable a simple vista en los estados financieros, a diferencia de las razones financieras (Beneish, 2001).

La gestión de ganancias, es definida como un proceso de tomar medidas deliberadas dentro de las limitaciones de los principios de contabilidad generalmente aceptados para lograr un nivel deseado de ganancias (Davidson, Stickney y Weil, 1987). El debate en relación a la gestión de ganancias, se centra en que es una práctica a discreción de la administración de las compañías que puede causar mala calidad de la información reportada por las empresas, atentar contra la transparencia de la misma y, por ende, no aportar la confianza necesaria a los grupos de interés de las empresas, lo cual puede acarrear problemas financieros en el futuro.

Por lo anterior, el presente estudio tiene como objetivo analizar la relación entre 
el riesgo de quiebra medido por el puntaje $\mathrm{Z}$ de Altman y la gestión de ganancias, cuyos efectos no se pueden observar en los estados financieros básicos a simple vista (e.g. estado de resultados integrales, estado de situación financiera, estado de flujo de efectivo). De esta manera esta investigación pretende medir el impacto de la discrecionalidad de los administradores del entorno empresarial mexicano y así, proporcionar una mejor perspectiva a académicos, reguladores, y grupos de interés de las empresas, al evaluar el grado de fiabilidad de sus estados financieros y su impacto en la solvencia empresarial.

Este documento se estructura de la siguiente forma. En la sección 2 se presenta la revisión de la literatura, así como los principales estudios en los que se relacionan la gestión de ganancias y el riesgo de quiebra en el mundo y en el contexto mexicano; en la sección 3 se explica la muestra a analizar, la forma en que se estimaron las variables y los modelos de panel de datos que se utilizaron; en la sección 4 se presentan los resultados de la presente investigación. Por último, en la sección 5 se presentan las conclusiones del estudio, así como las recomendaciones.

\section{Revisión de la literatura y desarrollo de hipótesis.}

Uno de los temas más investigados alrededor de las finanzas es la dificultad financiera (financial distress), la cual sus resultados pueden conducir a la quiebra de las empresas (Aruldoss, Travis y Venkatesan, 2015). Couwenberg (2015), menciona que la dificultad financiera se presenta en una empresa cuando su flujo de efectivo es incapaz de cumplir con sus obligaciones a corto plazo. Presentar dificultad financiera puede ser el comienzo para una empresa de estar en peligro de bancarrota en un futuro cercano, puesto que la bancarrota se asocia fuertemente con el nivel de insolvencia que pueda presentar una compañía en el largo plazo.

Martínez y Ronzón (2016), mencionan que en los últimos años ha tomado mucha importancia la medición del riesgo empresarial y su divulgación. Después de la crisis del 2008 $\square 2010$ quedó demostrado que la quiebra empresarial tiene implicaciones severas debido a que puede ocasionar un fuerte y negativo costo social, fracaso financiero, desempleo, así como aumentar la probabilidad de recesión $\mathrm{y}$, por ende, colocar en peligro a la economía en general (Bernanke, 1981; Aruldoss, et al., 2015; Mai, et al., 2019).

Con la finalidad de evitar el costo y las implicaciones que se mencionaron con anterioridad, las empresas pueden optar, de acuerdo a las regulaciones de cada país, por una figura legal para eludir la quiebra. Por ejemplo, en México esta figura es el concurso mercantil, mientras que en Estados Unidos es el Capítulo 11. Altman, Hotchkiss y Wang (2019), mencionan que un objetivo clave del capítulo 11 en E.U.A. o el concurso mercantil en México, es brindar la oportunidad, a las empresas que son más económicamente viables, de reorganizar sus activos y pasivos mientras liquida; y, a las empresas que no son económicamente viables, de recuperarse de la bancarrota.

A partir de los efectos financieros y económicos de las quiebras de empresas como Enron y WorldCom, se han observado señales de desconfianza e incertidumbre en los mercados de valores alrededor del mundo (Martínez y Ronzón, 2016). Para los investigadores, acreedores e inversionistas es de mucha importancia estudiar la predicción de quiebra empresarial, puesto que es un detonante principal para el riesgo crediticio y puede afectar a muchos grupos de interés (Mai, et al., 2019).

\subsection{Puntaje $Z$ de Altman}

La detección de dificultades financieras de las empresas es un tema que ha sido susceptible de análisis dentro del ámbito financiero (Altman, 1968). En busca de una herramienta útil para predecir las dificultades financieras de las empresas americanas, Altman (1968) publicó un estudio utilizando 
Cumpean Luna, Joel Alejandro; Briseño García, Arturo y Arango Herrera, Eduardo

Gestión de ganancias en el riesgo de quiebra de las empresas públicas mexicanas

información de 66 empresas manufactureras que cotizaban en una bolsa de valores, 33 en bancarrota y 33 sin dificultades financieras, en donde propone un modelo para conocer si se pudo prevenir la bancarrota de dichas empresas antes de que sucediera el evento. El modelo se desarrolló como sigue:

\section{$Z=0.012 \times 1+0.014 \times 2+0.033 \times 3+0.006 \times 4+0.999 \times 5$}

A lo largo de los años, muchas personas encontraron más conveniente redondear los valores como sigue, de acuerdo con Altman (2013):$$
Z=1.2 \times 1+1.4 \times 2+3.3 \times 3+0.6 \times 4+1.0 \times 5
$$

Donde: $\mathrm{x} 1=$ Capital de trabajo/total de activos; $\mathrm{x} 2$ = Utilidades retenidas/total de activos; $\mathrm{x} 3$ = EBIT/total de activos; $\mathrm{x} 4$ = Valor de mercado de la empresa/Valor total de la deuda en libros; $\mathrm{y}, \mathrm{x} 5=$ Ventas/total de activos.

Este primer modelo de Altman indicaba que si el puntaje $Z<1.81$, la empresa está en un riesgo inminente de bancarrota; si $Z>2.99$, se puede decir que la empresa goza de salud financiera; mientras que si $1.81<Z<2.99$, se considera que la empresa se encuentra en una 'zona gris', lo cual quiere decir que es factible que se enfrente a problemas de bancarrota durante los próximos 2 años.

Años después, Altman, Hartzell y Peck (1995) modificaron su modelo original enfocando dicha alteración para que sea útil y valido en mercados emergentes como el mercado mexicano. Dicho modelo fue desarrollado como sigue:

$$
Z=6.56 \times 1+3.26 \times 2+6.72 \times 3+1.05 \times 4+3.25
$$

Donde: $\mathrm{x} 1=$ Capital de trabajo/total de activos; $\mathrm{x} 2=$ Utilidades retenidas/total de activos; $\mathrm{x} 3=\mathrm{EBIT} /$ total de activos; $\mathrm{x} 4=$ Valor en libros del capital contable/pasivos totales; y, una constante de 3.25 , que permite estandarizar una calificación crediticia para dichos mercados emergentes (Altman, 2005).

Este último modelo, el cual denominaron como Puntaje para Mercados Emergentes (EMS score, por sus siglas en inglés), cambió sus intervalos de interpretación. En el EMS score, la 'zona inminente de bancarrota' es $Z<4.50$; la 'zona verde' es si $Z>5.86$; por último, la 'zona gris' cambia a $4.51<\mathrm{Z}<5.85$ (Altman, 2005).

Aunque el modelo de puntaje $\mathrm{Z}$ tiene muchos años que se desarrolló, aún se encuentra su uso de manera frecuente en múltiples situaciones gerenciales y sigue ayudando a tomar decisiones financieras, así como también le es útil a los inversionistas para tomar su decisión de inversión, bancos para otorgar créditos y auditores para evaluar la empresa a auditar (Altman, 2013; Bod'a y Úradníček, 2016; Fito, Plana-Erta y Llobet, 2018). 


\subsection{Gestión de ganancias}

Cómo se ha mencionado con anterioridad, la gestión de ganancias se ha estudiado desde diferentes puntos de vista, relacionándola con diferentes variables, y así mismo, se han creado varios conceptos alrededor de este término. A continuación, se muestra el Cuadro 1 con los conceptos más usados, desarrollados a lo largo de la literatura de gestión de ganancias.

\section{Cuadro 1}

\section{Definiciones de gestión de ganancias}

Autor(es)

Definición de gestión de ganancias.

\begin{abstract}
La gestión de ganancias ocurre cuando los gerentes utilizan el juicio en informes (Healy y Wahlen, 1999, financieros y en la estructuración de transacciones para alterar los informes financieros p.368) de la empresa o para influir en los resultados contractuales que dependen de los números contables informados.
\end{abstract}

Una intervención decidida en el proceso de información financiera externa, con la (Schipper, 1989, p.92) intención de obtener algún beneficio privado (en lugar de, digamos, simplemente facilitar el funcionamiento neutral del proceso).

La gestión de ganancias es vista como un proceso de tomar medidas deliberadas por parte (Davidson, et al., 1987) de los administradores para lograr un nivel deseado de ganancias reportadas, todo esto dentro del marco de los principios de contabilidad generalmente aceptados.
La gestión de ingresos se produce cuando los gerentes tienen un comportamiento discrecional relacionado con los números contables con o sin límites, y este comportamiento se puede adoptar para maximizar el valor de la empresa.

Fuente: Elaboración propia, 2020.

Tomando un consenso sobre varias de las definiciones anteriores, se puede decir que hay una gran probabilidad de que ocurra la gestión de ganancias cuando los gerentes manipulan los Devengos Discrecionales (DD) del total de los devengos (Watts y Zimmerman, 1978; Teoh, Welch y Wong, 1998; Xu, Taylor y Dugan, 2007). Por tal motivo, es de gran trascendencia que los modelos más comunes para la estimación de la gestión de ganancias incluyan los DD dentro de los mismos. Además, se puede notar que el análisis de la gestión de ganancia se enfoca mayormente en el uso discrecional de los devengos por parte de los administradores (Dechow, Sloan y Sweeney, 1995).
La técnica más común en la gestión de ganancias se da hacia aumentar el nivel de ingresos de la empresa en lugar de disminuirlos, todo en razón de reflejar un mayor valor y rendimiento por parte de la empresa, posiblemente para solicitar algún financiamiento o poder participar en algún mercado de valores (Beneish, 2001; Alkhabash y Al-Thuneibat, 2009); argumento que difiere con los hallazgos de Habib, Uddin e Islam (2013), puesto que ellos encontraron evidencia de que las empresas practican en su mayoría la gestión de ganancias encaminadas a disminuir las mismas.

Partiendo de lo anterior, se puede establecer que medir la gestión de ganancias 
no resulta sencillo puesto que no es un fenómeno observable a simple vista, por lo tanto, es menos probable que los inversionistas potenciales o los accionistas de una empresa puedan descubrir si la misma está empleando dicha práctica (Beneish, 2001). Esta situación genera un efecto dominó debido a que, si no se conoce el nivel de esta práctica en una empresa, el grado de inversión que se realice en una organización que gestiona sus ganancias será inapropiado, lo cual puede afectar hasta a la economía del país en el que cotiza (Linhares, Moraes y Beiruth, 2018). A México, como a los demás países con economías en crecimiento, le repercute en gran medida estas acciones puesto que de acuerdo con San Martín (2012), es muy probable que las empresas que cotizan en la BMV y que sean de alto crecimiento se involucren en la gestión de ganancias.

Healy (1985), realizó un aporte verdaderamente significativo al estudio de la gestión de ganancias, introduciendo los Ajustes por Devengo (AD) totales, que se utilizan para poder estimar dicho fenómeno y se pueden dividir en dos componentes: AD discrecionales y AD no discrecionales, siendo los primeros la clave para poder estimar la gestión de ganancias (Castillo y San Martín, 2008). Para poder medir los AD discrecionales (partidas no observables) se parte de los AD totales (Dechow, et al., 1995).

Es importante conocer lo que son los ajustes por devengo. El devengo es la principal función de los Principios de Contabilidad Generalmente Aceptados (PCGA), ahora Normas de Información Financiera (NIF), y es más común poder observar la gestión de ganancias en los devengos que en los componentes de flujo de efectivo (Beneish, 2001). Los AD se entienden como aquella parte de los ingresos o gastos que no necesariamente se realizan (se cobran o se pagan). Para los administradores resulta más complicado poder manipular los flujos de efectivo mediante tácticas contables, porque, para ello, es necesario realizar actividades reales (e.g. aumentar ventas, reducir gastos, retrasar o adelantar pagos, entre otros), no siendo así en los devengos, los cuales se generan producto de las normas contables y son más susceptibles a poder manipularlos (Alanís y Rodríguez, 2017).

En su aporte, Healy (1985) menciona que los ajustes por devengo totales (AT) se pueden obtener restándole al resultado contable (RC), el flujo de efectivo operativo (cash flow) (CFO) como se aprecia a continuación:

$$
A T=R C-C F O
$$

Este aporte es de suma importancia, pero posee la limitación de que no se puede generalizar para todos los países, debido a que no en todos se está obligado a emitir el estado de flujo de efectivo, por lo cual se ha establecido otra fórmula con la finalidad de poder obtener los ajustes por devengo totales con datos del balance general y el estado de resultados, e implica los ajustes por devengo discrecionales (AD) y por devengo no discrecionales (AND).

$$
A T=A D+A N D
$$

El modelo más utilizado en la literatura de estimación de gestión de ganancias ha sido el modelo modificado de Jones, propuesto por Dechow, et al. (1995) en donde ajustan a la variable de cambio en ventas con el cambio en cuentas por cobrar, con el fin de poder asumir la totalidad de las ventas a crédito como ajustes por devengo discrecionales, con lo que se proclama como un modelo superior al de Healy (1985); DeAngelo (1986) y Jones (1991), puesto que dichos modelos no toman en cuenta las condiciones económicas de la empresa (Castillo y San Martín, 2008).

\subsection{Gestión de ganancias y riesgo de quiebra}

Algunos estudios han intentado asociar la gestión de ganancias con el riesgo de quiebra. Por ejemplo, Kwak y Mo (2018) realizaron un estudio para medir el riesgo financiero de las empresas, usando el modelo de predicción del puntaje Z de Altman y su relación con la 
gestión de ganancias y los planes de pensión de los CEO's de las empresas. Sus resultados mostraron que los planes de pensión para ejecutivos juegan un papel muy importante dentro de las empresas, puesto que cuando los gerentes cuentan con compensaciones como planes de pensiones, tienden más a gestionar las ganancias hacia aumentar los ingresos durante el año en que se sufren dificultades financieras, sobre los gerentes que no cuentan con dichos planes.

Por otra parte, Ghazali, Shafie y Sanusi (2015) observan que los gerentes de las compañías se involucran en la gestión de ganancias cuando las finanzas de las mismas son saludables y su ganancia es alta, otros autores afirman que conocer el nivel de dificultad financiera que presentan las empresas es trascendente, puesto que afecta directamente la forma en que los administradores gestionan las ganancias. Entre más preocupación exista ante una posible quiebra, más se aplicará la gestión de ganancias hacia aumentar los ingresos (Rosner, 2003; Li, Abeysekera y Ma, 2011; Campa y Camacho-Miñano, 2014, 2015; Ahmadpour y Shahsavari, 2016).

En contraste, Charitou, Lambertides y Trigeorgis (2007) realizaron un estudio sobre el comportamiento de la gestión de ganancias de las empresas americanas con dificultades financieras en años previo a su quiebra. Sus resultados mostraron que las empresas gestionan sus ganancias hacia disminuirlas 1 año previo a la bancarrota.

En mercados emergentes como la India, también se ha estudiado la relación entre las dificultades financiera y la gestión de ganancias. Agrawal y Chatterjee (2015), realizaron un estudio con 150 empresas con dificultades financieras, utilizando como medida para gestión de ganancias, los devengos discrecionales; y, para dificultad financiera, utilizaron puntaje $\mathrm{Z}$ de Altman y el modelo "distancia al incumplimiento" de Merton, mejor conocido por sus siglas en inglés -DD model- (distance to default). Sus resultados mostraron una relación positiva y significativa de los modelos de predicción de quiebra con los devengos discrecionales que sirven para medir la gestión de ganancia. Mencionan que las empresas que tienen niveles altos tanto en puntaje $\mathrm{Z}$ de Altman como en el modelo DD indican una mejor salud financiera, las cuales, a su vez, practican una mayor gestión de ganancias. Es por ello que la hipótesis de la presente investigación está planteada como sigue:

H1.- La gestión de ganancias tiene un efecto positivo y significativo con el riesgo de quiebra de las empresas que cotizan en la BMV.

\section{Metodología}

En este estudio, se excluyeron las empresas del sector bancario y financiero para calcular las acumulaciones discrecionales siguiendo las recomendaciones descritas en la literatura (Kang y Sivaramakrishnan, 1995; Becker, et al., 1998). También se eliminaron las empresas con datos insuficientes para calcular las acumulaciones discrecionales.

De esta forma, de las 145 empresas que cotizaron en el periodo 2014 - 2018 en la BMV, se excluyeron un total de 59 empresas debido a 3 razones: (a) 25 se encontraban en el sector bancario o financiero, (b) 21 no se contaba con los datos necesarios para calcular la variable gestión de ganancias, y (c) 13 no cumplieron con el requisito de tener al menos 15 observaciones por año - industria (McVay, 2006; Barua, Lin y Sbaraglia, 2010; Zang, 2012; Farrell, Unlu y Yu, 2014), teniendo así una muestra de 86 empresas. Las industrias finales que conforman dicha muestra de 86 empresas son las siguientes: 1. Industrial, 2. Materiales, 3. Productos de consumo frecuente, y 4. Servicios y bienes de consumo no básico.

\subsection{Técnica de análisis y recolección de datos}

La presente investigación, es un estudio longitudinal mediante el uso del método de 
panel de datos en un periodo de 5 años, es decir, de los años 2014 al 2018. Este método consiste en una serie de tiempo por cada unidad u observación de una base de datos de corte transversal (Proaño, et al., 2019). Arellano y Bover (1990), identifican a un conjunto de datos panel cuando se tienen observaciones de series temporales sobre una muestra de unidades individuales. La característica fundamental de los datos de panel, que los distingue de las combinaciones de cortes transversales, es que durante un intervalo de tiempo se vigilan las mismas unidades (personas, empresas o condados) de un corte transversal (Wooldridge, 2009). Se utilizó el programa Stata versión 12 con la finalidad de correr la base de datos panel, el cual indica que la base está fuertemente balanceada.

La recopilación de información se realizó a través de bases de datos de Bloomberg. Respecto a la generación de la variable $\mathrm{Z}$ de Altman, se calculó de manera manual a partir de las partidas contables encontradas en las bases de datos mencionadas, y para la variable gestión de ganancias, se procedió a recolectar de las de Bloomberg para su cálculo.

Dado que, las bases de datos exploradas en Bloomberg, no contenían la información de todas las partidas de los 5 años para todas las empresas, se procedió a consultar los reportes anuales de las empresas publicadas en sus respectivas páginas web o en su defecto, en el portal de la BMV para rellenar los espacios vacíos en la base de datos.

\subsection{Definición y operacionalización de las variables}

\section{a. Variable dependiente}

Riesgo de quiebra: Couwenberg (2015), menciona que la dificultad financiera se presenta en una empresa cuando su flujo de efectivo es incapaz de cumplir con sus obligaciones a corto plazo. Presentar dificultad financiera puede ser el comienzo para una empresa de estar en peligro de bancarrota en un futuro cercano, puesto que la bancarrota se asocia fuertemente con el nivel de insolvencia que pueda presentar una compañía. Se utilizó el modelo de Altman, et al. (1995) con el fin de medir el riesgo de quiebra que presenten las empresas de la BMV. Dicho modelo y sus variaciones han sido la herramienta más utilizada para medir el riesgo de quiebra en la literatura (Abreu y Morales, 2008; Bryan, et al., 2013).

El modelo de Altman, et al. (1995), está compuesto de varias razones financieras con la finalidad de medir el nivel de solvencia, liquidez, rentabilidad, entre otros. El modelo está desarrollado cómo sigue:

\section{$Z=6.56 \times 1+3.26 \times 2+6.72 \times 3+1.05 x 4+3.25$}

\section{b. Variable independiente}

Gestión de Ganancias: Ocurre cuando los gerentes utilizan su juicio en la presentación de informes financieros para influir en los resultados contractuales que dependen de los números contables informados (Healy y Wahlen, 1999). En ese sentido, el valor de gestión de ganancias, está representado en valor absoluto debido a que la hipótesis de investigación no predice ninguna dirección específica para la gestión de ganancias. Además, el valor absoluto también captura las reversiones de acumulación tras la gestión de ganancias (Becker, et al., 1998; Klein, 2002; Chen, Elder y Hsieh, 2007; Cohen, Dey y Lys, 2008). Su cálculo se realiza de acuerdo a las siguientes fórmulas:

1. Cálculo de los devengos totales, usando la fórmula establecida en Dechow, et al. (1995): 


$$
D T=(\Delta A . C .-\Delta P . C .-\Delta e f v o+\Delta D . C . P .-D e p \& A m o r t) / A \cdot T \cdot t-1
$$

2. Estimación de los parámetros específicos de las empresas:

$$
D T_{t}=a_{1}\left(\frac{1}{A_{t-1}}\right)+a_{2}\left(\Delta \operatorname{Vtas}_{t}\right)+a_{3}\left(P P E_{t}\right)+v_{t}
$$

3. Los parámetros específicos de las empresas que se obtuvieron en el paso 2, se introducen en el modelo de Dechow et al. (1995):

$$
D N D_{\tau}=\alpha_{1}\left(\frac{1}{A_{\tau-1}}\right)+\alpha_{2}\left(\Delta \operatorname{tas}_{\tau}-\Delta C x C_{\tau}\right)+\alpha_{3}\left(P P E_{\tau}\right)
$$

4. Por último, los devengos discrecionales son calculados por diferencia con los datos que ya se obtuvieron en el paso número $1 \mathrm{y}$ paso número 3 :

$$
\text { D. D. }{ }_{\tau}=\text { D.T. } \tau-D . N . D_{\tau}
$$

\section{c. Variables de control}

\begin{tabular}{|c|c|c|}
\hline Variable & Definición & Medición \\
\hline $\begin{array}{l}\text { Apalancamiento } \\
\text { financiero }\end{array}$ & $\begin{array}{l}\text { Anggraeni y Wardhani (2017), mencionan que el } \\
\text { apalancamiento es la relación que existe de deuda a activos, } \\
\text { que se adquieren de las deudas totales divididas entre los } \\
\text { activos totales. }\end{array}$ & Deuda a largo plazo / Activo total \\
\hline ROA & $\begin{array}{l}\text { Es una razón financiera utilizada para medir la } \\
\text { rentabilidad de la empresa respecto a sus activos totales, } \\
\text { la cual se calcula dividiendo el resultado antes de partidas } \\
\text { extraordinarias de la empresa i en el año t (Lin, Lo y Wu, } \\
\text { 2016; Anagnostopoulou y Tsekrekos, 2017). }\end{array}$ & EBIT / Activo total \\
\hline Diversificación & $\begin{array}{l}\text { Es la estrategia empresarial utilizada actualmente por las } \\
\text { empresas para mantener presencia en más de un sector } \\
\text { económico. }\end{array}$ & Índice Herfindahl \\
\hline Tamaño & $\begin{array}{l}\text { El tamaño se puede observar en el valor de los activos o } \\
\text { ventas totales con los que cuenta la empresa. Regularmente } \\
\text { se utiliza el logaritmo natural de los activos totales para } \\
\text { controlar el efecto del tamaño de la empresa (Habib, et al., } \\
\text { 2013; Lin, et al., 2016). }\end{array}$ & Ln de Activos totales \\
\hline Q de Tobin & $\begin{array}{l}\text { La Q de Tobin pretende marcar un panorama respecto al } \\
\text { valor que posee una empresa. }\end{array}$ & $\frac{\text { Valor de la empresa en el mercado }}{\text { Valor de la empresa en libros }}$ \\
\hline Industria & $\begin{array}{l}\text { Se utilizaron variables dummies para controlar el efecto } \\
\text { que tiene el pertenecer a cierta industria. }\end{array}$ & $\begin{array}{l}1 \text { cuando pertenece a cierta industria, } \\
0 \text { cuando no. }\end{array}$ \\
\hline Años & $\begin{array}{l}\text { Se incluyeron variables dummies para controlar el efecto de } \\
\text { los años en que cotizaron las empresas en la BMV para el } \\
\text { periodo } 2014-2018 \text {. }\end{array}$ & $\begin{array}{l}1 \text { cuando cotizó en cierto año, } 0 \\
\text { cuando no. }\end{array}$ \\
\hline
\end{tabular}

Cuadro 2.

\section{Cuadro 2}

\section{Operacionalización de variables de control}

Fuente: Elaboración propia, 2020. 


\subsection{Modelo econométrico}

Para el contraste de la hipótesis, donde se planteó una relación negativa y significativa entre el riesgo de quiebra y la gestión de ganancias practicada por las empresas de la BMV, se construyó el siguiente modelo econométrico:

$R Q_{i t}=\beta_{0 i}+\beta_{1} G G_{i t}+\beta_{2}$ Controles $_{i t}+\mu_{i t}$

Donde: $\mathrm{RQ}=$ Riesgo de quiebra de la empresa $\mathrm{i}$ en el año $\mathrm{t}$; $\mathrm{GG}=$ Devengos discrecionales que son la medida para observar la gestión de ganancias de la empresa i en el año t.

Dicho modelo pretende explicar la relación entre gestión de ganancias y riesgo de quiebra medida con el puntaje $\mathrm{Z}$ de Altman, así como la forma en que se ve influenciado el puntaje $Z$ en función de la gestión de ganancias.

\section{a. Tratamiento y robustez del modelo de regresión panel}

El presente estudio utiliza la técnica de análisis de datos de panel, para ello fue necesario la depuración de observaciones en el periodo del 2014 al 2018 debido a la naturaleza de los sectores. En este sentido, para dar paso a la generación de resultados, la base de datos fue sometida a diferentes análisis de robustez con el objetivo de corroborar que el modelo que se propone cumple con los supuestos de heterocedasticidad y no autocorrelación serial.

Como se mencionó anteriormente, el modelo econométrico panel fue sometido a la prueba Hausman, para distinguir entre efectos aleatorios y efectos fijos. El resultado de la prueba fue que se identificó como mejor modelo de estimación en panel de datos, el de efectos fijos con un resultado Hausman de X2 $=131.09, \mathrm{p}=0.0000$.

Posteriormente, se consideraron análisis para heterocedasticidad (Breusch y Pagan, 1980) y autocorrelación serial (Wooldridge, 2009), encontrando ambos problemas presentes en el modelo, X2 $=10.55, \mathrm{p}=$ 0.0006 para heterocedasticidad y $\mathrm{F}=20.378$, $\mathrm{p}=0.0000$ en autocorrelación serial. Para corregir esta situación fue necesario el uso del método GLS (Generalized Least Square), conocido en la literatura como panel dinámico y permitiendo el tratamiento de los problemas antes mencionados.

\section{Resultados y discusión}

La Tabla 1, muestra los principales estadísticos descriptivos y correlaciones de las variables presentes en el estudio. La variable con mayores observaciones fue gestión de ganancias $(G G)$ con 430 observaciones; mientras que la variable que tuvo menos observaciones fue la variable $\mathrm{Q}$ de Tobin $(Q T o b)$ con 370 observaciones. Todas las correlaciones son menores a \pm 0.4 lo cual es esperado y deseado. Esto demuestra que no hay problemas de multicolinealidad entre las variables.

\section{Tabla 1}

Estadísticos descriptivos y coeficientes de correlación

\begin{tabular}{|c|c|c|c|c|c|c|c|c|c|c|c|c|}
\hline 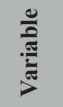 & $\hat{0}$ & 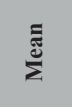 & $\begin{array}{l}\dot{\Xi} \\
\dot{\Xi} \\
\dot{0}\end{array}$ & 主 & $\sum_{\Sigma}^{\star}$ & $\underset{\simeq}{\stackrel{O}{\prime \prime}}$ & ن & $\Xi$ & $\underset{\varrho}{\nwarrow}$ & 音 & Е & $\frac{0}{0}$ \\
\hline RQ & 426 & 7.15 & 13.84 & -72.57 & 181.13 & 1 & & & & & & \\
\hline GG & 430 & 0.06 & 0.10 & 0.00007 & 1.39 & -0.15 & 1 & & & & & \\
\hline AFin & 419 & 21.06 & 14.85 & 0.00 & 80.50 & -0.15 & -0.14 & 1 & & & & \\
\hline ROA & 416 & 4.02 & 13.26 & -132.87 & 124.52 & 0.03 & 0.29 & -0.25 & 1 & & & \\
\hline
\end{tabular}




\section{Cont... Tabla 1}

\begin{tabular}{ccccccccccccc}
\hline Div & 415 & 6346.9 & 2442.2 & 1466.1 & 10000 & 0.09 & -0.08 & -0.01 & 0.05 & 1 & \\
Tam & 411 & 23.82 & 1.69 & 8.33 & 27.10 & 0.02 & -0.11 & 0.14 & -0.01 & -0.08 & 1 \\
Qtob & 370 & 2.34 & 2.45 & 0.13 & 20.06 & 0.04 & -0.06 & 0.33 & 0.13 & 0.12 & 0.18 & 1 \\
\hline
\end{tabular}

Fuente: Elaboración propia, 2020.

Después de obtener los estadísticos descriptivos y las correlaciones entre las variables, se procedió a realizar una regresión panel utilizando 3 métodos de modelación, es decir, el de Mínimos Cuadrados Ordinaros Acumulados (Pooled), el de Datos Panel de Efectos Fijos, y por último, el método de Mínimos Cuadrados Generalizados (GLS) por sus siglas en inglés. La Tabla 2, muestra los resultados de los tres modelos que se analizaron.

Tabla 2

Resumen de los modelos

\begin{tabular}{|c|c|c|c|c|c|c|c|c|c|}
\hline \multicolumn{10}{|c|}{ Análisis $2014-2018$} \\
\hline \multicolumn{3}{|c|}{ Observaciones: 361} & \multicolumn{4}{|c|}{ Observaciones: 361} & Obs: 361 & \multicolumn{2}{|c|}{$\mathrm{N}^{\circ}$ de grupos: 81} \\
\hline & \multicolumn{2}{|c|}{ Modelo 1} & & \multicolumn{3}{|c|}{ Modelo 2} & \multicolumn{3}{|l|}{ Modelo 3} \\
\hline & \multicolumn{2}{|c|}{$\mathrm{F}=3.15$} & & \multicolumn{3}{|c|}{ Wald chi $(13)=18.35$} & \multicolumn{3}{|c|}{ Wald chi $(13)=42.65$} \\
\hline & \multicolumn{2}{|c|}{$($ Prob $>F)=0.0002$} & & \multicolumn{3}{|c|}{$\left(\right.$ Prob $\left.>\mathrm{chi}^{2}\right)=0.1447$} & \multicolumn{3}{|c|}{$\left(\right.$ Prob $\left.>\mathrm{chi}^{2}\right)=0.0001$} \\
\hline & \multicolumn{6}{|c|}{$\mathrm{R}^{2}=0.0718$} & & & \\
\hline Var. Dep. & \multicolumn{2}{|c|}{ Modelo 1} & & \multicolumn{3}{|c|}{ Modelo 2} & \multicolumn{3}{|l|}{ Modelo 3} \\
\hline RQ & $\beta$ & S.E. & $\mathrm{P}>|\mathrm{z}|$ & $\beta$ & S.E. & $\mathrm{P}>|\mathrm{z}|$ & $\beta$ & S.E. & $\mathrm{P}>|\mathrm{z}|$ \\
\hline GG & 14.135 & 4.674 & $* * *$ & 4.88 & 4.255 & & 14.135 & 4.583 & $* * *$ \\
\hline Afin & -0.125 & 0.033 & $* * *$ & -0.081 & 0.042 & $*$ & -0.125 & 0.032 & $* * *$ \\
\hline ROA & 0.006 & 0.044 & & 0.044 & 0.040 & & 0.006 & 0.042 & \\
\hline Div & 0.000 & 0.000 & & 0.000 & 0.000 & & 0.000 & 0.000 & $*$ \\
\hline Tam & 0.401 & 0.328 & & 0.282 & 0.558 & & 0.401 & 0.321 & \\
\hline Qtob & 0.376 & 0.196 & & 0.163 & 0.258 & & 0.376 & 0.192 & $*$ \\
\hline S_Indus & 1.566 & 1.406 & & -0.192 & 2.358 & & 0.577 & 1.246 & $* *$ \\
\hline S_Mat & 4.022 & 1.443 & $* * *$ & 4.409 & 2.429 & $*$ & 3.033 & 1.281 & $*$ \\
\hline S_Prod_cons & (omitte & & & -0.175 & 2.723 & & -0.989 & 1.466 & \\
\hline S_Ser_bien_nb & 0.989 & 1.496 & & (omitted & & & (omitted) & & \\
\hline y2014 & (omitte & & & -0.243 & 1.097 & & -0.534 & 1.365 & \\
\hline y2015 & 0.176 & 1.369 & & -0.892 & 1.082 & & -0.357 & 1.349 & \\
\hline
\end{tabular}




\section{Cont... Tabla 2}

\begin{tabular}{lllllll}
\hline y2016 & 2.286 & 1.349 & 1.203 & 1.034 & 1.752 & 1.314 \\
y2017 & -0.052 & 1.361 & -0.288 & 1.050 & -0.586 & 1.337 \\
y2018 & 0.534 & 1.393 & (omitted) & & (omitted) & \\
Constante & -5.123 & 8.732 & -1.524 & 13.768 & -3.599 & 8.136 \\
\hline
\end{tabular}

Nota: $* * * \mathrm{p}<0.01, * * \mathrm{p}<0.05, * \mathrm{p}<0.1$

Para el análisis de regresión se decidió aplicar una transformación a la variable RQ utilizando una codificación inversa (reverse scoring). De esta forma, valores altos del puntaje de Z Altman representan un mayor riesgo de quiebra, mientras que valores bajos de la variable representa un menor riesgo de quiebra para las empresas. Esta transformación permite interpretar de una mejor manera la relación entre las variables GG y RQ.

Fuente: Elaboración propia, 2020.

Los resultados expuestos en la Tabla 2, muestran de manera evolutiva los efectos de las variables establecidas en el modelo econométrico. Primeramente, se muestra un análisis pooled en el modelo 1, consecuentemente se presenta el modelo 2, donde se someten los resultados a través de la técnica de datos de panel mediante el método de estimación de efectos fijos, y finalmente, en el modelo 3 se establecen los resultados mediante el método GLS. De acuerdo a lo anterior, a continuación, se explican cada uno de los modelos aquí mencionados.

El modelo $1(\mathrm{~F}=3.15, \mathrm{R} 2=7.18 \%, \mathrm{p}$ $>\mathrm{F}=0.0002$ ), muestra una significancia muy alta y un valor de R2 alto para el tamaño de la muestra y las variables independientes estudiadas, de acuerdo con Hair, et al. (1999). En dicho modelo, se puede observar un efecto positivo y significativo de la gestión de ganancias en la variable riesgo de quiebra $(\beta 1=14.135, \mathrm{p}>=0.003)$.

De igual manera, el modelo 2 (Wald Chi $=18.35$, y p $>$ chi $\left.^{2}=0.1447\right)$, presenta un efecto positivo de la gestión de ganancias sobre la variable riesgo de quiebra, similar al modelo 1, sin embargo, dicho efecto es no significativo $\left(\beta 1=4.88, \mathrm{p}>\mathrm{chi}^{2}=0.251\right)$.

Finalmente, el modelo 3 (Wald X2 $=42.65$ y $\mathrm{P}=0.0001$ ) cuyo análisis se realizó mediante el método GLS el cual evita problemas de heteroscedasticidad $y$ autocorrelación serial, presenta igual que los modelos anteriores, un efecto positivo de la variable de gestión de ganancias sobre el riesgo de quiebra $(\beta 1=14.135)$ y con una significatividad alta $(\mathrm{p}>0.002)$, mostrando así que, a mayor gestión de ganancias, se reduce el valor para la variable $(R Q) \mathrm{y}$, por ende, el riesgo de quiebra de las empresas aumenta, por lo que no existe evidencia para rechazar $\mathrm{H} 1$.

\section{Conclusiones}

El presente estudio tiene como objetivo analizar la relación entre el riesgo de quiebra medido por el puntaje $\mathrm{Z}$ de Altman y la gestión de ganancias estimada por la parte discrecional de los devengos totales de las empresas, cuyos efectos no se pueden observar en los estados financieros básicos a simple vista (e.g. Estado de Resultados Integrales, Estado de Situación Financiera, Estado de Flujo de Efectivo). De esta manera, este estudio pretende medir el impacto de la discrecionalidad de los administradores del entorno empresarial mexicano y así, proporcionar una mejor perspectiva a académicos, reguladores y grupos de interés de las empresas, al evaluar el potencial impacto de la información financiera expresada en sus Estados Financieros y su influencia en la solvencia empresarial, es decir, su riesgo de quiebra.

Los resultados del presente estudio muestran que existe un efecto positivo y 
significativo entre las variables de estudio, por lo que el aumento en la gestión de ganancias incrementa el riesgo de quiebra, motivo por el cual, se acepta la hipótesis planteada, la cual propone que la gestión de ganancias tiene un efecto positivo y significativo con el riesgo de quiebra de las empresas que cotizan en la BMV.

De igual manera, los hallazgos de esta investigación se consideran de gran importancia para la comunidad académica y empresarial, sobre todo para el contexto mexicano, puesto que indica que cuando los administradores utilizan su discreción en las normas contables para manipular la información financiera presentada en los estados financieros de las empresas, puede generar efectos negativos, aun cuando la intención de los administradores pueda tener un beneficio de corto plazo, como disuadir a sus acreedores de capital, reguladores o al gobierno, en realidad están afectando negativamente a la empresa, debido a que las hace más propensas de que no puedan cumplir con sus obligaciones de retribuir a sus acreedores de capitales y se encuentren en un riesgo de quiebra en el mediano y largo plazo.

Debido a que la gestión de ganancias es vista como una práctica con falta de ética, los administradores de las empresas deben de resistirse a la incitación de realizar dicha actividad, puesto que, al presentar información financiera con falta de calidad y transparencia, pueden ocasionar que los diversos grupos de interés de las organizaciones mantengan una postura incrédula sobre los estados financieros y además puede tener implicaciones en los mercados de capitales.

Este trabajo tiene áreas de oportunidad, pues resulta necesario indagar más sobre el tema de riesgo de quiebra, pero en especial sobre la gestión de ganancias para el contexto empresarial mexicano y latinoamericano, puesto que la mayoría de los estudios realizados sobre el tema han sido por parte de autores norteamericanos, británicos y asiáticos, los mismos que cuentan en su mayoría con bases de datos mucho más completas así como extensas de las que se cuentan en Latinoamérica, las cuales también tienen el problema de que los periodos de tiempo son más estrechos.

Por otra parte, otra limitante que tiene el presente estudio es que únicamente se utilizó un modelo basado en el devengo. Futuras investigaciones pueden aportar a la literatura en el contexto mexicano, utilizando otros modelos de devengo como el modelo de Kothari, así como el de gestión de ganancias real, introducido por Roychowdhury, con el fin de observar la comparabilidad entre los principales modelos para gestión de ganancias de acuerdo a la literatura consultada. Para riesgo de quiebra, también se puede agregar otros modelos como el de Ohlson, el cual además del modelo del puntaje $\mathrm{Z}$ de Altman, es ampliamente aceptado para financial distress y bankruptcy risk (riesgo de quiebra).

Finalmente, es importante comentar la importancia que tienen los administradores financieros y los auditores, puesto que en ellos recae la responsabilidad de la divulgación de la información financiera generada por las empresas. No deben de permitir que los administradores utilicen su discreción y juicio sobre los momentos contables de las NIF para manipular los estados financieros que informan a diversos grupos de interés sobre el desempeño de las empresas, debido a que esto genera problemas a la hora de tomar decisiones y afectar el capital invertido y prestado a las empresas. La ética, es un valor que debe de ir inherente a toda profesión y en el mundo empresarial no es la excepción.

\section{Referencias bibliográficas}

Abreu, M., y Morales, J. A. (2008). Las empresas con problemas en la Bolsa Mexicana de Valores (BMV). Denarius, (16), 139-174.

Agrawal, K., y Chatterjee, C. (2015). Earnings management and financial distress: Evidence from India. Global Business Review, 16, 140-154. https://doi. org/10.1177/0972150915601928

Ahmadpour, A., y Shahsavari, M. (2016). 
Cumpean Luna, Joel Alejandro; Briseño García, Arturo y Arango Herrera, Eduardo

Gestión de ganancias en el riesgo de quiebra de las empresas públicas mexicanas

Earnings management and the effect of earnings quality in relation to bankruptcy level (firms listed at the tehran stock exchange). Iranian Journal of Management Studies (IJMS), 9(1), 77-99. https://doi. org/10.22059/IJMS.2016.55036

Alanís, I., y Rodríguez, M. D. P. (2017). La práctica de la gestión de ganancias. Innovaciones de Negocios, 14(28), 189-198.

Al-khabash, A. A., y Al-Thuneibat, A. A. (2009). Earnings management practices from the perspective of external and internal auditors: Evidence from jordan. Managerial Auditing Journal, 24(1), 58-80. https:// doi.org/10.1108/02686900910919901

Altman, E. I. (1968). Financial ratios, discriminant analysis and the prediction of corporate bankruptcy. The Journal of Finance, 23(4), 589-609. https:// doi.org/10.1111/j.1540-6261.1968. tb00843.x

Altman, E. I. (2005). An emerging market credit scoring system for corporate bonds. Emerging Markets Review, 6(4), 311-323. https://doi.org/https://doi. org/10.1016/j.ememar.2005.09.007

Altman, E. I. (2013). Predicting financial distress of companies: revisiting the Z-Score and ZETA ${ }^{\circledR}$ models. In A. R. Bell, C. Brooks y M. Prokopczuk (Eds.), Handbook of research methods and applications in empirical finance (pp. 428-456). Edward Elgar Publishing.

Altman, E. I., Hartzell, J., y Peck, M. (1995). Emerging market corporate bonds - a scoring system. In R. M. Levich (Ed.), Emerging market capital flows: Proceedings of a Conference held at the Stern School of Business, New York University on May 23-24, 1996 (pp. 391-400). Springer. https://doi. org/10.1007/978-1-4615-6197-2 25
Altman, E. I., Hotchkiss, E., y Wang, W. (2019). Corporate financial distress, restructuring, and bankruptcy: Analyze leveraged finance, distressed debt, and bankruptcy (4th ed). Wiley Finance.

Anagnostopoulou, S. C., y Tsekrekos, A. E. (2017). The effect of financial leverage on real and accrual-based earnings management. Accounting and Business Research, 47(2), 191236. https://doi.org/10.1080/0001478 $\underline{8.2016 .1204217}$

Anggraeni, M. D., y Wardhani, R. (2017). The effect of leverage and IFRS convergence on earnings management through real activities manipulation in Asia. Asian Journal of Business and Accounting, 10(1), 87-125.

Arellano, M., y Bover, O. (1990). La econometría de datos panel. Investigaciones Económicas (Segunda época), XIV(1), 3-45.

Aruldoss, M., Travis, M. L., y Venkatesan, V. P. (2015). A reference model for business intelligence to predict bankruptcy. Journal of Enterprise Information Management, 28(2), 186-217. https:// doi.org/10.1108/JEIM-09-2013-0069

Báez, M. D. P., y Puentes, G. A. (2018). Parámetros financieros para la toma de decisiones en pequeñas y medianas empresas del municipio Duitama, Colombia. Revista de Ciencias Sociales (Ve), XXIV(1), 67-84.

Barua, A., Lin, S., y Sbaraglia, A. M. (2010). Earnings management using discontinued operations. The Accounting Review, 85(5), 1485 1509. https://doi.org/10.2308/ accr.2010.85.5.1485

Becker, C. L., DeFond, M. L., Jiambalvo, J., y Subramanyam, K. R. (1998). The Effect of Audit Quality on Earnings Management. 
Contemporary Accounting Research, 15(1), 1-24. https://doi. org/10.1111/j.1911-3846.1998. tb00547.x

Beneish, M. D. (2001). Earnings management: A perspective. Managerial Finance, 27(12), 3-17. https://doi. org/10.1108/03074350110767411

Bernanke, B. (1981). Bankruptcy, liquidity, and recession. The American Economic Review, 71(2), 155-159.

Bod'a, M., y Úradníček, V. (2016). The portability of altman's Z-score model to predicting corporate financial distress of Slovak companies. Technological and Economic Development of Economy, 22(4), 532-553. https://doi. org/10.3846/20294913.2016.1197165

Breusch, T. S., y Pagan, A. R. (1980). The lagrange multiplier test and its applications to model specification in econometrics. The Review of Economic Studies, 47(1), 239-253. https://doi.org/10.2307/2297111

Bryan, D., Dinesh, G., y Tripathy, A. (2013). Bankruptcy risk, productivity and firm strategy. Review of Accounting and Finance, 12(4), 309-326. https://doi. org/10.1108/RAF-06-2012-0052

Campa, D., y Camacho-Miñano, M-D-M. (2014). Manipulación del resultado entre empresas concursadas no cotizadas: Evidencia en España. Revista Española de Financiacion y Contabilidad, 43(1), 3-20. https://doi. org/10.1080/02102412.2014.890820

Campa, D., y Camacho-Miñano, M-D-M. (2015). The impact of SME's pre-bankruptcy financial distress on earnings management tools. International Review of Financial Analysis, 42, 222-234. https://doi. org/10.1016/j.irfa.2015.07.004

Castillo, L. A., y San Martín, J. M. (2008). Los ajustes por devengo como medida de discrecionalidad directiva. Una revisión bibliográfica. Contaduría y Administración, (226), 9-37. $\quad$ https://doi.org/10.22201/ fca.24488410e.2008.641

Charitou, A., Lambertides, N., y Trigeorgis, L. (2007). Managerial discretion in distressed firms. The British Accounting Review, 39(4), 323$346 . \quad$ https://doi.org/10.1016/j. bar.2007.08.003

Chen, K. Y., Elder, R. J., y Hsieh, Y-M. (2007). Corporate governance and earnings management: The implications of corporate governance best-practice principles for taiwanese listed companies. Journal of Contemporary Accounting \& Economics, 3(2), 73105. https://doi.org/10.1016/S18155669(10)70024-2

Cohen, D. A., Dey, A., y Lys, T. Z. (2008). Real and accrual-based earnings management in the pre - and post - sarbanes - oxley periods. The Accounting Review, 83(3), 757$787 . \quad \mathrm{https} / / /$ doi.org/10.2308/ accr.2008.83.3.757

Couwenberg, O. (2015). Financial distress. Wiley Encyclopedia of Management, 1-3. https://doi. org/10.1002/9781118785317. weom040039

Davidson, S., Stickney, C. P., y Weil, R. L. (1987). Accounting: The language of business ( 7 th ed). T. Horton.

DeAngelo, L. E. (1986). Accounting numbers as market valuation substitutes: A study of management buyouts of public stockholders. The Accounting Review, 61(3), 400-420.

Dechow, P. M., Sloan, R. G., y Sweeney, A. P. (1995). Detecting earnings management. The Accounting Review, 70(2), 193-225.

Farrell, K., Unlu, E., y Yu, J. (2014). 
Cumpean Luna, Joel Alejandro; Briseño García, Arturo y Arango Herrera, Eduardo

Gestión de ganancias en el riesgo de quiebra de las empresas públicas mexicanas

Stock repurchases as an earnings management mechanism: The impact of financing constraints. Journal of Corporate Finance, 25, 1-15. https://doi.org/10.1016/j. jcorpfin.2013.10.004

Fito, M. A., Plana-Erta, D., y Llobet, J. (2018). Usefulness of $\mathrm{Z}$ scoring models in the early detection of financial problems in bankrupt Spanish companies. Intangible Capital, 14(1), 162-170. https://doi.org/10.3926/ic.1108

Ghazali, A. W., Shafie, N. A., y Sanusi, Z. M. (2015). Earnings management: An analysis of opportunistic behaviour, monitoring mechanism and financial distress. Procedia Economics and Finance, 28, 190-201. https://doi. org/10.1016/S2212-5671(15)01100-4

Habib, A., Uddin, M. B., e Islam, A. (2013). Financial distress, earnings management and market pricing of accruals during the global financial crisis. Managerial Finance, 39(2), 155-180. https://doi. org/10.1108/03074351311294007

Hair, J. F., Black, W. C., Babin, B. J., Anderson, R. E., y Tatham, R. L. (1999). Multivariate data analysis. Prentice Hall.

Healy, P. M. (1985). The effect of bonus schemes on accounting decisions. Journal of Accounting and Economics, 7(1-3), 85-107. https://doi. org/10.1016/0165-4101(85)90029-1

Healy, P. M., y Wahlen, J. M. (1999). A review of the earnings management literature and its implications for standard setting. SSRN Electronic Journal, 13(4), 365-383. https://doi. org/10.2139/ssrn. 156445

Jones, J. J. (1991). Earnings management during import relief investigations. Journal of Accounting Research, 29(2), 193-228. https://doi. org/10.2307/2491047

Kang, S-H., y Sivaramakrishnan, K. (1995). Issues in testing earnings management and an instrumental variable approach. Journal of Accounting Research, 33(2), 353-367. https://doi. org/10.2307/2491492

Klein, A. (2002). Audit committee, board of director characteristics, and earnings management. Journal of Accounting and Economics, 33(3), 375-400. https://doi.org/10.1016/S0165$\underline{\text { 4101(02)00059-9 }}$

Kwak, B., y Mo, K. (2018). Executive pension, default risk, and earnings management*. Asia-Pacific Journal of Accounting and Economics, 25(3-4), 463-480. https://doi.org/10.1080/1608 $\underline{1625.2016 .1277951}$

Li, F., Abeysekera, I., y Ma, S. (2011). Earnings management and the effect of earnings quality in relation to stress level and bankruptcy level of Chinese listed firms. Corporate Ownership and Control, 9(1), 366-391. https://doi. org/10.22495/cocv9i1c3art2

Lin, H-W. W., Lo, H-C., y Wu, R-S. (2016). Modeling default prediction with earnings management. PacificBasin Finance Journal, 40(B), 306-322. https://doi.org/10.1016/j. pacfin.2016.01.005

Linhares, F. S., Moraes, F., y Beiruth, A. X. (2018). Earnings management and investment efficiency. Review of Business Management, 20(2), 295310. https://doi.org/10.7819/rbgn. v20i2.3180

Mai, F., Tian, S., Lee, C., y Ma, L. (2019). Deep learning models for bankruptcy prediction using textual disclosures. European Journal of Operational Research, 274(2), 743-758. https://doi. org/10.1016/j.ejor.2018.10.024

Martínez, H. R., Cazallo, A. M., Meñaca, I., 
y Uribe, C. M. (2020). Desempeño financiero de las empresas minoristas de alimentos y bebidas en Barranquilla - Colombia. Revista de Ciencias Sociales (Ve), XXVI(1), 144160. $\quad$ https://doi.org/10.31876/rcs. v26i1.31316

Martínez, R., \& Ronzón, X. (2016). Risk disclosure: First overview in the Mexican context. World of Accounting Science, 18, 511-536. https://bit.ly/3oRaPUFMcVay, S. E. (2006). Earnings management using classification shifting: An examination of core earnings and special items. The Accounting Review, 81(3), 501-531. $\quad$ https://doi.org/10.2308/ accr.2006.81.3.501

Proaño, S. A., Quiñonez, E. S., Molina, C. J., \& Mejía, O. G. (2019). Desarrollo económico local en Ecuador: Relación entre producto interno bruto y sectores económicos. Revista de Ciencias Sociales (Ve), XXV(E-1), 82-98. https:// doi.org/10.31876/rcs.v25i1.29598

Rodríguez, M., Piñeiro, C., y de Llano, P. (2014). Determinación del riesgo de fracaso financiero mediante la utilización de modelos paramétricos, de inteligencia artificial, y de información de auditoría. Estudios de Economía, 41(2), 187-217. https://doi.org/10.4067/S0718$\underline{52862014000200002}$

Rosner, R. L. (2003). Earnings manipulation in failing firms. Contemporary Accounting Research, 20(2), 361-408.
https://doi.org/10.1506/8EVN-9KRB3AE4-EE81

San Martín, J. M. (2012). An empirical examination of ownership structure, earnings management and growth opportunities in mexican market. International Journal of Business and Social Research, 2(7), 103-123.

Schipper, K. (1989). Comentary on earnings management. Accounting Horizons, 3(4), 91-102.

Teoh, S. H., Welch, I., y Wong, T. J. (1998). Earnings management and the underperformance of seasoned equity offerings. Journal of Financial Economics, 50(1), 63-99. https://doi. org/10.1016/S0304-405X(98)00032-4

Watts, R. L., y Zimmerman, J. L. (1978). Towards a positive theory of the determination of accounting standards. The Accounting Review, 53(1), 112134.

Wooldridge, J. (2009). Introductory econometrics: A modern approach. Cengage Learning.

Xu, Z. R., Taylor, G. K., y Dugan, M. T. (2007). Review of real earnings management literature. Journal of Accounting Literature, 26, 195-228.

Zang, A. Y. (2012). Evidence on the trade-off between real activities manipulation and accrual-based earnings management. The Accounting Review, 87(2), 675-703. https://doi. org/10.2308/accr-10196 